\title{
Recovery of Sensorimotor Function after Experimental Stroke Correlates with Restoration of Resting-State Interhemispheric Functional Connectivity
}

\author{
Maurits P. A. van Meer, ${ }^{1,2}$ Kajo van der Marel, ${ }^{1}$ Kun Wang, ${ }^{3}$ Willem M. Otte, ${ }^{1}$ Soufian el Bouazati, ${ }^{1}$ Tom A. P. Roeling, ${ }^{4}$ \\ Max A. Viergever, ${ }^{1}$ Jan Willem Berkelbach van der Sprenkel, ${ }^{2}$ and Rick M. Dijkhuizen ${ }^{1}$ \\ ${ }^{1}$ Biomedical Magnetic Resonance Imaging and Spectroscopy Group, Image Sciences Institute, University Medical Center Utrecht, 3584 CX Utrecht, The \\ Netherlands, ${ }^{2}$ Department of Neurosurgery, Rudolf Magnus Institute of Neuroscience, University Medical Center Utrecht, 3584 CX Utrecht, The \\ Netherlands, ${ }^{3}$ National Laboratory of Pattern Recognition, Institute of Automation, Chinese Academy of Sciences, Beijing 100190, China, and ${ }^{4}$ Department \\ of Anatomy, Division of Surgical Specialties, University Medical Center Utrecht, 3584 CX Utrecht, The Netherlands
}

Despite the success of functional imaging to map changes in brain activation patterns after stroke, spatiotemporal dynamics of cerebral reorganization in correlation with behavioral recovery remain incompletely characterized. Here, we applied resting-state functional magnetic resonance imaging (rs-fMRI) together with behavioral testing to longitudinally assess functional connectivity within neuronal networks, in relation to changes in associated function after unilateral stroke in rats. Our specific goals were (1) to identify temporal alterations in functional connectivity within the bilateral cortical sensorimotor system and (2) to elucidate the relationship between those alterations and changes in sensorimotor function. Our study revealed considerable loss of functional connectivity between ipsilesional and contralesional primary sensorimotor cortex regions, alongside significant sensorimotor function deficits in the first days after stroke. The interhemispheric functional connectivity restored in the following weeks, but remained significantly reduced up to 10 weeks after stroke in animals with lesions that comprised subcortical and cortical tissue, whereas transcallosal neuroanatomical connections were preserved. Intrahemispheric functional connectivity between primary somatosensory and motor cortex areas was preserved in the lesion border zone and moderately enhanced contralesionally. The temporal pattern of changes in functional connectivity between bilateral primary motor and somatosensory cortices correlated significantly with the evolution of sensorimotor function scores. Our study (1) demonstrates that poststroke loss and recovery of sensorimotor function is associated with acute deterioration and subsequent retrieval of interhemispheric functional connectivity within the sensorimotor system and (2) underscores the potential of rs-fMRI to assess spatiotemporal characteristics of functional brain reorganization that may underlie behavioral recovery after brain injury.

\section{Introduction}

Stroke is one of the main causes of disability worldwide. Nevertheless, acute neurological deficits may partially resolve at later stages. Spontaneous restitution of lost function after stroke has been associated with brain plasticity, which refers to the ability of the brain to compensate for loss of function through reorganization of neuronal networks. Functional and structural brain reorganization after stroke has been demonstrated in several animal and human studies (for review, see Nudo, 1999; Kreisel et al., 2006). In recent years, neuroimaging modalities, in particular functional magnetic resonance imaging (fMRI), have been successfully applied to in vivo, whole-brain assessment of changes in

Received Nov. 17, 2009; revised Dec. 30, 2009; accepted Jan. 23, 2010.

This work was supported by the Alexandre Suerman Program of the University Medical Center Utrecht (M.P.A.v.M.), a Utrecht University Short Stay Fellowship (K.W.), and Utrecht University's High Potential Program (R.M.D.). We thank Annette van der Toorn and Jan-Willem de Groot for technical assistance.

Correspondence should be addressed to either Dr. Maurits P. A. van Meer or Dr. Rick M. Dijkhuizen, Image Sciences Institute, University Medical Center Utrecht, Heidelberglaan 100, 3584 CX Utrecht, The Netherlands. E-mail: maurits@invivonmr.uu.nl or rick@invivonmr.uu.nl.

DOI:10.1523/JNEUROSCI.5709-09.2010

Copyright $\odot 2010$ the authors $\quad 0270-6474 / 10 / 303964-09 \$ 15.00 / 0$ functional activation patterns in stroke patients (Cramer and Bastings, 2000; Rijntjes and Weiller, 2002; Calautti and Baron, 2003) and experimental stroke models (Dijkhuizen et al., 2001, 2003; Weber et al., 2008). Such fMRI papers have reported loss of activation responses in the ipsilesional hemisphere, sometimes combined with enhanced contralesional activation, at early stages after unilateral stroke, whereas reinstatement of activity in ipsilesional regions has been frequently observed at later stages. In previous studies, we observed widespread contralesional activation in response to stimulation of the impaired limb at acute and subacute time points after stroke in rats with large lesions involving cortical and subcortical tissue (Dijkhuizen et al., 2001, 2003). Functional recovery was mainly associated with the preservation or restoration of normal unilateral ipsilesional activation (Dijkhuizen et al., 2001, 2003; Weber et al., 2008). Positron emission tomography (PET) and fMRI studies in stroke patients have also demonstrated that improved motor recovery goes together with enhanced activity of the remaining intact ipsilesional motor network, whereas sustained involvement of contralesional motor regions is associated with poor recovery (for review, see Calautti and Baron, 2003). Nevertheless, the precise spatiotemporal pat- 
tern of reorganization within specific neural networks in relation to recovery of related functions remains mostly unresolved.

Poststroke fMRI studies have been mostly confined to detection of explicit task- or stimulus-induced activation responses in selective functional areas. An alternative, relatively new fMRI approach [i.e., resting-state fMRI (rs-fMRI)] may allow more extensive assessment of changes in organization of whole functional networks (Fox and Raichle, 2007; Auer, 2008). rs-fMRI aims to detect baseline brain activity related to ongoing neuronal signaling at "rest" and is performed by low-pass filtering of spontaneous blood oxygenation level-dependent (BOLD) fMRI signals (Biswal et al., 1995). Analogous to PET (Horwitz et al., 1984) and electrophysiological measurements (Engel et al., 1991) of clear temporal correlation between neuronal activity in connected brain areas under resting-state conditions, synchronization of low-frequency $(<0.1 \mathrm{~Hz})$ BOLD signal fluctuations between different brain regions is believed to reflect functional connectivity. Validation studies have shown that significant temporal correlation of low-frequency BOLD oscillations is particularly found in regions with strong neuroanatomical connections (Vincent et al., 2007; Honey et al., 2009). Moreover, simultaneous rs-fMRI and electroencephalogram recordings have revealed that synchronized low-frequency BOLD signals within functional networks are associated with distinct electrophysiological signatures (Laufs et al., 2003; Lu et al., 2007; He et al., 2008; Shmuel and Leopold, 2008). Since rs-fMRI does not require specific stimulation or active task execution, it allows arbitrary assessment of functional connectivity within various neuronal networks in a single experiment. Hence, rs-fMRI provides an ideal tool to study the dynamics of changes in organization of functional networks in diseased brain under relatively stable and noninvasive conditions.

The goal of our study was to characterize the dynamics of functional connectivity changes within the bilateral sensorimotor network of rats recovering from experimental stroke. We hypothesized that loss and recovery of sensorimotor functions correlate with weakening and strengthening of functional connectivity between certain sensorimotor regions in the brain. To that aim, we conducted serial rs-fMRI along with repeated behavioral testing after unilateral stroke in rats, and searched for potential correlations between the pattern of functional connectivity changes within the sensorimotor system and loss and recovery of sensorimotor function.

\section{Materials and Methods}

Stroke model. All animal procedures were approved by the Utrecht University Ethical Committee on Animal Experiments, and experiments were performed in accordance with the guidelines of the European Communities Council Directive.

A total of 17 male Wistar rats, weighing 250-280 g, was included in the study. Rats were anesthetized with $4 \%$ isoflurane for endotracheal intubation, followed by mechanical ventilation with $2.0 \%$ isoflurane in air/ $\mathrm{O}_{2}(2: 1)$. Blood oxygen saturation and heart rate were continuously monitored during surgical procedures. Body temperature was maintained at $37.0 \pm 0.5^{\circ} \mathrm{C}$.

Transient focal cerebral ischemia was induced by $90 \mathrm{~min}$ occlusion of the right middle cerebral artery (tMCA-O) with an intraluminal filament (Longa et al., 1989). In brief, a 4.0 polypropylene suture (Ethicon) with a silicon-coated tip (tip diameter of $\sim 0.4 \mathrm{~mm}$ ) was introduced into the right external carotid artery and advanced through the internal carotid artery until a slight resistance was felt, indicating that the MCA was occluded. After $90 \mathrm{~min}$, the filament was withdrawn from the internal carotid artery to allow reperfusion. We have previously demonstrated significant hypoperfusion and successful subsequent reperfusion, as well as substantial ischemic tissue damage with this intraluminal filament model (Dijkhuizen et al., 1997, 2003).
During surgery, a total of $5 \mathrm{ml}$ of saline was administered by subcutaneous injection to compensate for loss of water and minerals. Before and $8 \mathrm{~h}$ after surgery, rats received a subcutaneous injection of $0.03 \mathrm{mg} / \mathrm{kg}$ buprenorphine (Temgesic; Schering-Plough) for postsurgical pain relief.

Behavioral tests. Two behavioral tests were used to measure sensorimotor function. First, six different motor, sensory, and tactile tests were applied (van der Zijden et al., 2008a), which provided an overall sensorimotor performance score (SPS) on a scale of 0 to -20 points, with -20 as maximum deficit score. These tests included measurement of spontaneous activity during $1 \mathrm{~min}$, gait disturbances such as circling toward the paretic side, degree or absence of pathological postural reflexes when held by the tail, degree of asymmetry in resistance between left and right lateral push, degree of asymmetry in grasping strength onto a rod between left and right forepaw, and degree or absence of forelimb placing onto a table rim after stimulation of the vibrissae. Second, an adhesive removal test was performed (Schallert et al., 2000). A small circular sticky tape (Herma Labels) with a diameter of $12 \mathrm{~mm}$ was attached to the distal radial region of the wrist of the left or right forelimb, and the removal time (in seconds) was measured for each forelimb with a maximally allowed removal time of $60 \mathrm{~s}$. During $3 \mathrm{~d}$ before tMCA-O, rats were trained to remove adhesives from the left or right forepaw within $1 \mathrm{~s}$.

SPS and adhesive removal time difference (from baseline measurement) from the affected left and unaffected right forepaw were scored a few hours before (=day 0$)$ and at $1,3,7,14,21,28,42,56$, and $70 \mathrm{~d}$ after stroke.

Structural and functional MRI. Structural MRI and rs-fMRI measurements were done at $2 \mathrm{~d}$ before tMCA-O $(=$ day -2$)$ and at $3,7,21$ and $70 \mathrm{~d}$ after stroke.

After behavioral testing, rats were anesthetized with $4 \%$ isoflurane for endotracheal intubation, followed by mechanical ventilation with $1.8 \%$ isoflurane in air $/ \mathrm{O}_{2}$ (2:1) during MRI experiments (ventilation rate, 52-59 $\mathrm{min}^{-1}$ ). MRI measurements were conducted on a $4.7 \mathrm{~T}$ horizontal bore MR system (Varian) with use of a Helmholtz volume coil $(90 \mathrm{~mm}$ diameter) and an inductively coupled surface coil (25 $\mathrm{mm}$ diameter) for signal excitation and detection, respectively. Rats were placed in a MRcompatible stereotactic holder and immobilized with earplugs and a tooth holder. During MRI, blood oxygen saturation and heart rate were continuously monitored by a pulse oximeter (8600V; Nonin Medical) with the probe positioned on a hindpaw. In addition, expired $\mathrm{CO}_{2}$ was continuously monitored with a capnograph (Multinex 4200; Datascope Corporation), and body temperature was maintained at $37.0 \pm 0.5^{\circ} \mathrm{C}$ using a feedback-controlled heating pad. End-tidal $\mathrm{CO}_{2}$ levels were kept within the normal range, equivalent to arterial $\mathrm{pCO}_{2}$ levels between 35 and $45 \mathrm{mmHg}$ (calculated from previous calibration measurements), by adjusting ventilation volume and/or rate.

First, multiecho multislice $\mathrm{T}_{2}$-weighted MRI [repetition time (TR)/ echo time (TE), 3000/17.5 ms; echo train length, 8 ; 19 coronal slices; field of view (FOV), $32 \times 32 \mathrm{~mm}^{2}$; acquisition matrix, $128 \times 128$; voxel resolution, $0.25 \times 0.25 \times 1.0 \mathrm{~mm}^{3}$ ] was conducted to assess ischemic lesion size and location. Then, for at least $15 \mathrm{~min}$, end-tidal isoflurane was reduced to $1 \%$. At this level of isoflurane anesthesia, coupling of cerebral glucose utilization and cerebral blood flow is maintained (Maekawa et al., 1986; Lenz et al., 1998). Furthermore, in other experiments we found preservation of coherence of low-frequency BOLD signal fluctuations between bilateral homologous sensorimotor regions at 0.5 and $1.0 \%$ isoflurane, which diminished at $2.9 \%$ isoflurane (our unpublished data). Subsequently, rs-fMRI was conducted during $10 \mathrm{~min}$ with a $\mathrm{T}_{2}{ }^{*}$-weighted gradient echo echo planar imaging sequence $\left(50^{\circ}\right.$ flip angle; TR/TE, 1000/19 ms; 13 coronal slices; FOV, $32 \times 32 \mathrm{~mm}^{2}$; acquisition matrix, $64 \times 64$; voxel resolution, $0.5 \times 0.5 \times 1.0 \mathrm{~mm}^{3}$; 600 BOLD images).

Neuronal tract tracing. In a subset of animals, we conducted neuronal tract tracing with manganese-enhanced MRI (van der Zijden et al., 2007) or dual immunohistochemical staining of biotinylated dextran amine (BDA) and Phaseolus vulgaris leucoagglutinin (PHA-L), after the structural and functional MRI experiments.

First, for manganese-enhanced MRI, gradient echo $\mathrm{T}_{1}$-weighted MRI (TR/TE, $60-6000 / 2.5 \mathrm{~ms} ; 19$ coronal slices; FOV, $32 \times 32 \mathrm{~mm}^{2}$; acquisition matrix, $128 \times 128$; voxel resolution, $0.25 \times 0.25 \times 1.0 \mathrm{~mm}^{3}$ ) was 
conducted to obtain baseline measurements of tissue $R_{1}\left(1 / T_{1}\right)$ at $70 \mathrm{~d}$ after stroke (directly after the final rs-fMRI session).

At $72 \mathrm{~d}$ after tMCA-O, animals were anesthetized by subcutaneous injection of a mixture of $0.55 \mathrm{mg} / \mathrm{kg}$ midazolam (Dormicum; Roche) and $0.315 \mathrm{mg} / \mathrm{ml}$ fentanylcitrate and $10 \mathrm{mg} / \mathrm{ml}$ fluanisone $(0.55 \mathrm{mg} / \mathrm{kg})$ (Hypnorm; VetaPharma) and positioned in a stereotactic holder with earplugs and a tooth holder. Blood oxygen saturation and heart rate were continuously monitored, and body temperature was maintained at $37.0 \pm 0.5^{\circ} \mathrm{C}$. For manganese-enhanced MRI, rats received an injection of $0.2 \mu \mathrm{l}$ of $1 \mathrm{M} \mathrm{MnCl}_{2}$ in PBS (infusion rate, $0.05 \mu \mathrm{l} / \mathrm{min}$ ) into the contralesional primary motor cortex (M1) at $0.5 \mathrm{~mm}$ anterior and 2.5 $\mathrm{mm}$ lateral to bregma, and $1.5 \mathrm{~mm}$ below the dura mater as described by van der Zijden et al. (2007). Another group of rats received 10\% BDA $(10,000$ molecular weight; lysine fixable; Invitrogen) in $0.01 \mathrm{~m} \mathrm{PBS,} \mathrm{pH}$ 7.4, and 5\% PHA-L (Vector Laboratories) in $0.05 \mathrm{~m}$ Tris-buffered saline (TBS) in the contralesional M1 by iontophoresis at $0.0 \mathrm{~mm}$ anterior and $2.0 \mathrm{~mm}$ lateral to bregma for BDA, and at $2.0 \mathrm{~mm}$ anterior and $3.0 \mathrm{~mm}$ lateral to bregma for PHA-L. Therefore, a glass micropipette with an internal tip diameter of $\sim 40 \mu \mathrm{m}$ was lowered at $1.5 \mathrm{~mm}$ below the dura mater, followed by $7 \mathrm{~s}$ on/off pulsed positive $10 \mu \mathrm{A}$ DC currents for 15 min [adapted from the study by Dolleman-Van der Weel et al. (1994)].

At $2 \mathrm{~d}$ after manganese injection, in vivo postcontrast $\mathrm{T}_{1}$-weighted MRI was done as described above. At $13 \mathrm{~d}$ after administration of BDA and PHA-L, rats were killed by an intraperitoneal injection of $120 \mathrm{mg} / \mathrm{kg}$ pentobarbital (Euthanimal; Alfasan). Transcardial perfusion-fixation was performed promptly by infusion with $180 \mathrm{ml}$ of PBS followed by 180 $\mathrm{ml}$ of $4 \%$ paraformaldehyde in $0.1 \mathrm{M}$ PBS. At $24 \mathrm{~h}$ after fixation in $4 \%$ paraformaldehyde at $4^{\circ} \mathrm{C}$, brain tissue was immersed in $30 \%$ sucrose in $0.05 \mathrm{M}$ PBS and $0.05 \%$ sodium azide until equilibrium. Coronal sections of 40-60 $\mu \mathrm{m}$ thickness were cut frozen on a sliding microtome and collected in 0.05 m PBS with $30 \%$ sucrose and $0.05 \%$ sodium azide. Subsequently, incubations were conducted on free-floating sections under gentle agitation at room temperature. First, for labeling of PHA-L, sections were rinsed (three times for $10 \mathrm{~min}$ each time in $0.05 \mathrm{~m}$ TBS at $\mathrm{pH} 7.6$ ) and incubated for $30 \mathrm{~min}$ in $3 \% \mathrm{H}_{2} \mathrm{O}_{2}$ in TBS, followed by $1 \mathrm{~h}$ preincubation in $5 \%$ normal goat serum (NGS) in TBS containing $0.1 \%$ Triton X-100 (TBS-TX). Subsequently, sections were incubated overnight in rabbit anti-PHA-L (Dako Denmark) (1:2000 in TBS-TX, 0.1\% NGS, and $0.1 \%$ bovine serum albumin). After rinsing, sections were incubated in biotinylated goat-anti-rabbit (Dako Denmark) (1:1000 in TBS-TX and 1\% NGS) for $1 \mathrm{~h}$. This allowed dual association of immunolabeled PHA-L and BDA with avidin-biotin peroxidase complex (ABC) (Dako Denmark), followed by reaction with nickel-enhanced diaminobenzidine tetrahydrochloride (DAB-Ni) (Sigma-Aldrich) to enhance staining. The steps for the DAB-Ni staining included the following: (1) rinsing in TBS three times for 10 min each time; (2) 60 min incubation in ABC; (3) rinsing in TBS two times for 8 min each time; (4) rinsing in $0.01 \mathrm{~m}$ sodium acetate, $\mathrm{pH} 6.5$, two times for $8 \mathrm{~min}$ each time; (5) $10 \mathrm{~min}$ incubation in DAB-Ni solution ( $1 \mathrm{ml}$ of $4 \mathrm{mg}$ of $\mathrm{DAB}$ in $10 \mathrm{ml}$ of Tris- $\mathrm{HCl}$ buffer, $8 \mathrm{ml}$ of $0.01 \mathrm{M}$ sodium acetate, $1 \mathrm{ml}$ of $3 \%$ nickel ammonium sulfate, and $8.3 \mu \mathrm{l}$ of $30 \% \mathrm{H}_{2} \mathrm{O}_{2}$ ); (6) rinsing in TBS four times for 8 min each time; (7) overnight air drying, dehydration, and coverslipping with Entallan (Merck). Whole-brain photographs (using a Sony 3 CCD-Color Video Camera) of stained sections were made under light microscopy (Leica Microsystems) for qualitative assessment.

Experimental groups. Inclusion and group assignments were based on SPS and adhesive removal times at day 1 after stroke, and size and location of ischemic lesions on $\mathrm{T}_{2}$-weighted images at day 3 after stroke, respectively. Based on previous findings, we excluded animals with SPS above -2 or adhesive removal times within $10 \mathrm{~s}$ at day 1 after stroke because of minor stroke injury $(n=3)$. Animals with only a subcortical lesion on $\mathrm{T}_{2}$-weighted images at day 3 were assigned in group I $(n=5)$, whereas animals with a lesion involving both subcortical and cortical tissue were assigned in group II $(n=9)$. A subset of animals in groups I $(n=2 \times 1$ for manganese-enhanced MRI and BDA/PHA-L staining, respectively) and II ( $n=2 \times 2$ for manganeseenhanced MRI and BDA/PHA-L staining, respectively) was used for neuronal tract tracing.
Data analysis. Motion correction with FLIRT (FMRIB FSL; www. fmrib.ox.ac.uk/fsl) and spatial smoothing (with a $1 \mathrm{~mm}$ full width at half-maximum Gaussian kernel) were applied on the rs-fMRI time series. Nonphysiological artifacts and physiological noise in the BOLD fMRI signals were minimized by several steps of linear regression before low bandpass filtering, in accordance with a recent study by Weissenbacher et al. (2009). Linear regressions were applied against the rigid-body realignment parameters, white matter signals from the internal capsule, CSF signals from the lateral ventricles, and the global mean signal of all voxels within a brain mask. Inclusion of these computed regressors in the analysis of rs-fMRI signals minimizes potential BOLD fluctuations of non-neuronal origin and thereby increases the specificity of neuronal functional connectivity measurements (Fox et al., 2009; Weissenbacher et al., 2009). Subsequently, low-frequency BOLD fluctuations were extracted by applying a bandpass filter with $0.01<f<0.1 \mathrm{~Hz}$ (Cordes et al., 2001; Pawela et al., 2008), using AFNI software (R. W. Cox, Bethesda, MD; http://afni. nimh.nih.gov/afni). For functional connectivity analysis, the first and last $50 \mathrm{~s}$ of BOLD fMRI acquisition were discarded to exclude saturation effects and bandpass filter-induced artifacts, respectively. Functional connectivity was measured as the correlation coefficient $r$ between low-frequency BOLD fluctuations in different voxels or regions of interest (ROIs) outside the lesion area. For variance stabilization, $r$ was Fisher-transformed according to $z^{\prime}=\ln ((1+r) /(1-r)) / 2$. Whole-brain functional connectivity maps were obtained by voxelwise calculation of $z^{\prime}$ with the mean time series signal from a seed region as reference. Brain voxels inside the ischemic lesion were excluded by masking out the lesion, as delineated on $\mathrm{T}_{2}$ maps at $3 \mathrm{~d}$ after stroke [segmentation tool, FSL FAST (http://www.fmrib.ox.ac.uk/fsl)]. Group mean functional connectivity maps, overlaid on an anatomical brain template, were obtained by averaging across subjects.

For quantification of functional connectivity between specific functional fields, we calculated $z^{\prime}$ between mean signals of selected ROIs. To this end, $\mathrm{T}_{2}$-weighted MR images were registered with an affine transformation to a MRI dataset of a reference brain, which was matched to a three-dimensional model of a rat brain atlas (Paxinos and Watson, 2005). Bilateral ROIs were selected within the cortical sensorimotor network according to the stereotaxic coordinates of the atlas and included the forelimb region of the primary somatosensory cortex (S1fl) and M1 (see Fig. 1). We also selected a nonsensorimotor region [i.e., the primary visual cortex (V1)]. ROIs were projected onto the multislice rs-fMRI time series. Interhemispheric functional connectivity was calculated between bilateral homologous ROIs. In addition, intrahemispheric functional connectivity was calculated between $\mathrm{S} 1 \mathrm{fl}$ and $\mathrm{M} 1$ in both the right (ipsilesional) and left (contralesional) hemisphere.

Premanganese and postmanganese $\mathrm{R}_{1}$ maps were registered to the rat brain atlas in the same way as described above. $\Delta \mathrm{R}_{1}$ maps were computed by subtracting premanganese from postmanganese $R_{1}$ maps. To assess transhemispheric neuronal tracer transport from the injection site (i.e., contralesional $\mathrm{M} 1$ ), we measured manganese-induced $\Delta \mathrm{R}_{1}$ in the corpus callosum (CC) and ipsilesional M1 and S1fl (ROIs selected according to the stereotaxic coordinates of the atlas).

Statistics. Linear mixed-model analysis was performed on sensorimotor function scores and functional connectivity between groups and time points. The factors group, time, and interaction (group by time) were set as fixed effects, and subject (rat) was set as random effect. Post hoc Bonferroni's correction was used to test all pairwise comparisons between groups and time points per group.

Linear mixed-model analysis was also used to calculate correlation between sensorimotor function and interhemispheric functional connectivity. After normalization based on SDs, standardized regression coefficients between normalized behavioral scores (SPS and adhesive removal time difference) and normalized interhemispheric $z^{\prime}$ values (for bilateral S1fl, M1, and V1) were calculated by linear regression.

SPSS software (version 16.0; SPSS) was used for all statistical analyses. Values are shown as mean $\pm \mathrm{SD}$. A value of $p<0.05$ was considered significant. 


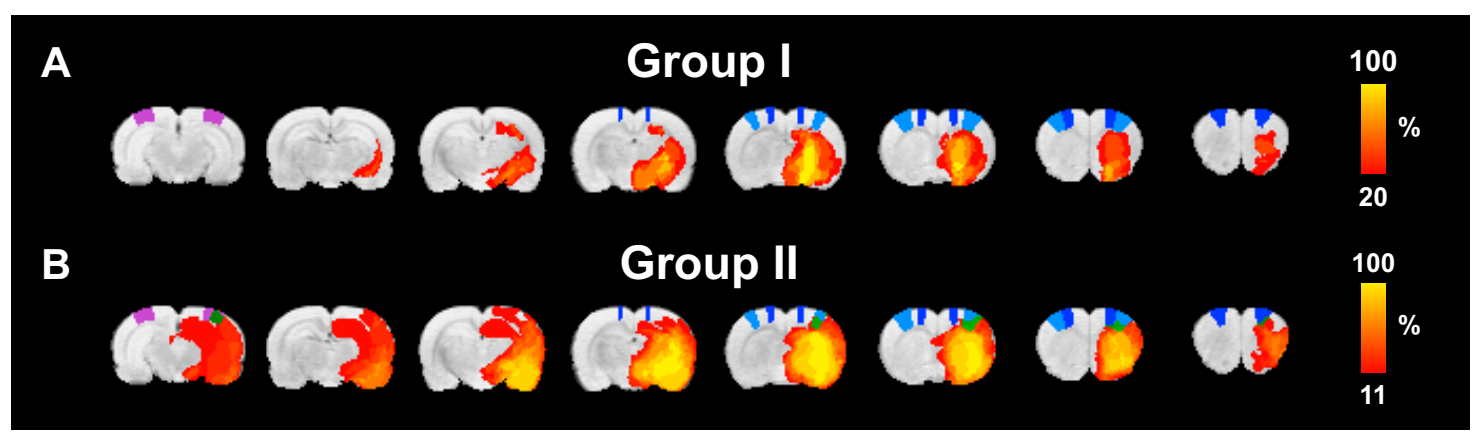

Figure 1. Lesion incidence maps. $A, B$, Color-coded local incidence of $\mathrm{T}_{2}$-based lesion, from 20 to $100 \%$ for group I $(n=5)(\boldsymbol{A})$ and from 11 to $100 \%$ for group II $(n=9)(\boldsymbol{B})$, overlaid on consecutive coronal rat brain slices from a $\mathrm{T}_{2}$-weighted template. Lesion areas with clear $\mathrm{T}_{2}$ prolongation were manually outlined on $\mathrm{T}_{2}$ maps at $3 \mathrm{~d}$ after stroke. The ROls M1 (primary motor cortex) (dark blue), S1fl (forelimb region of the primary somatosensory cortex) (light blue), and V1 (primary visual cortex) (purple) are depicted on the template. The green areas in group Il are voxels with lesioned tissue that overlap with $\mathrm{S} 1 \mathrm{fl}$ and $\mathrm{V} 1$ regions $(\boldsymbol{B})$ and were excluded from the functional connectivity analysis by previous lesion segmentation.
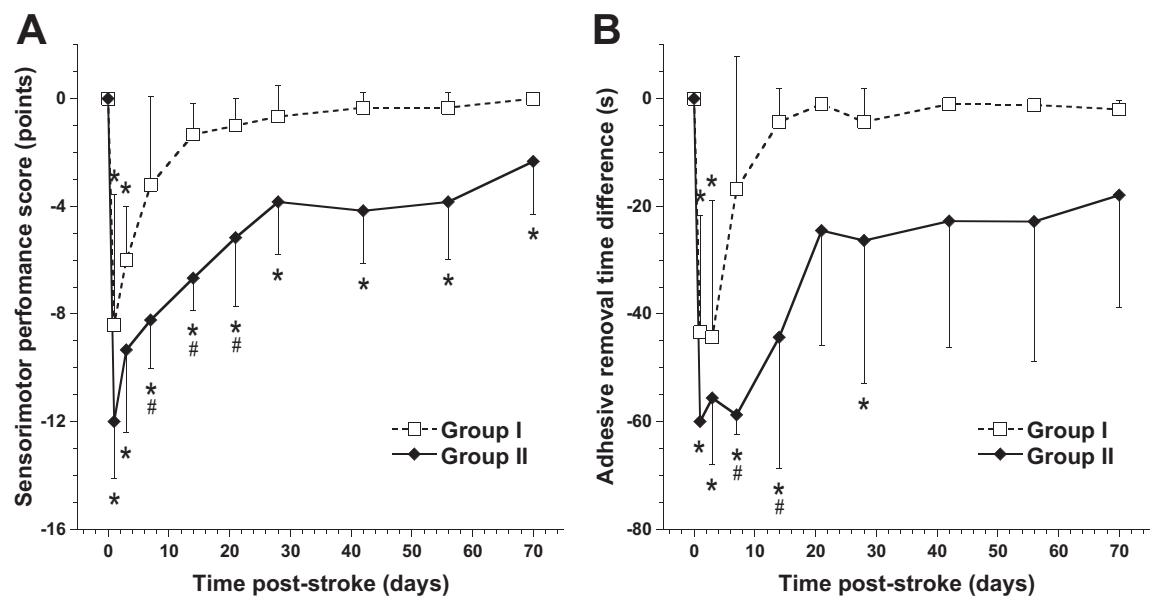

Figure 2. Time course of sensorimotor functions. $\boldsymbol{A}, \boldsymbol{B}$, Mean $( \pm$ SD) SPS (in points from 0 to -20$)(\boldsymbol{A})$ and time difference of adhesive removal from left affected forelimb (in seconds) $(\boldsymbol{B})$ at different time points after tMCA-0 for groups I (dotted lines) and II (solid lines). ${ }^{*} p<0.05$ versus pre; ${ }^{p} p<0.05$ versus group I.

\section{Results}

\section{Stroke lesion}

$\mathrm{T}_{2}$ maps at $3 \mathrm{~d}$ after tMCA-O showed clear hyperintensity in the subcortical caudate-putamen in five animals (group I), whereas nine animals demonstrated prolonged $\mathrm{T}_{2}$ in both subcortical (caudate-putamen) and cortical tissue (primary and secondary somatosensory cortices) (group II) (see incidence maps of manually outlined lesions on $\mathrm{T}_{2}$ maps at $3 \mathrm{~d}$ after stroke in Fig. 1).

\section{Sensorimotor function}

Time courses of the SPS and adhesive removal time difference are depicted in Figure 2, $A$ and $B$, respectively. Sensorimotor function was significantly reduced in both groups in the first $3 \mathrm{~d}$ after stroke (low SPS and large time difference) and recovered thereafter. After $7 \mathrm{~d}$ after stroke, SPS and adhesive removal time were not significantly different from baseline in group I animals. Adhesive removal time in group II animals had normalized from day 42 onward, whereas SPS showed a similar pattern of recovery but remained significantly lowered. Except for an initial delay early after surgery at poststroke day 1 in group II, no significant change in adhesive removal time difference was measured when the adhesive was applied to the right, unimpaired forelimb.

\section{Functional connectivity}

Figure $3, A$ and $B$, shows mean functional connectivity maps for groups I and II, respectively. The structurally intact right, ipsilesional S1fl was chosen as seed ROI. Two days before stroke, both groups demonstrated significant correlation of the time courses of low-frequency BOLD signals (i.e., strong functional connectivity) between right S1fl (seed region) and other sensorimotor cortical areas (e.g., primary and secondary motor cortices, forelimb and hindlimb regions of the primary somatosensory cortex, and secondary somatosensory cortex) in both hemispheres. Similar results were found with right $\mathrm{M} 1$ as seed ROI (supplemental Fig. 1, available at www.jneurosci.org as supplemental material).

At all time points after tMCA-O, intrahemispheric functional connectivity of right, ipsilesional S1fl with M1 remained intact in both groups. Furthermore, there was no significant difference in this intrahemispheric functional connectivity between time points, for the ipsilesional (Fig. $4 A$ ) as well as contralesional hemisphere (Fig. 4B). However, a significant group effect was found in contralesional intrahemispheric functional connectivity between S1fl and M1, which was higher in group II than in group I $(F=4.84 ; p=0.033)$.

Interhemispheric functional connectivity between the right, ipsilesional S1fl and left, contralesional sensorimotor cortex was diminished at 3 and $7 \mathrm{~d}$ after stroke in group I animals (Fig. $3 A$ ), and at 3, 7, and $21 \mathrm{~d}$ after stroke in group II animals (Fig. 3B). Moreover, negative correlations were found in the contralesional sensorimotor cortex of group II animals (Fig. 3B). Similar findings were observed on functional connectivity maps with right, ipsilesional M1 as seed ROI (supplemental Fig. 1, available at www.jneurosci.org as supplemental material).

Figure 5 shows the temporal profile of interhemispheric connectivity between right and left homologous ROIs. At $3 \mathrm{~d}$ after stroke, the loss of interhemispheric functional connectivity was particularly evident for the sensorimotor ROIs S1fl (Fig. $5 A$ ) and M1 (Fig. 5B) in group II animals, where $z^{\prime}$ was significantly reduced compared with prestroke and compared with group I animals. Interhemispheric functional connectivity between the right and left $\mathrm{V} 1$ areas was also significantly reduced at $3 \mathrm{~d}$ after 

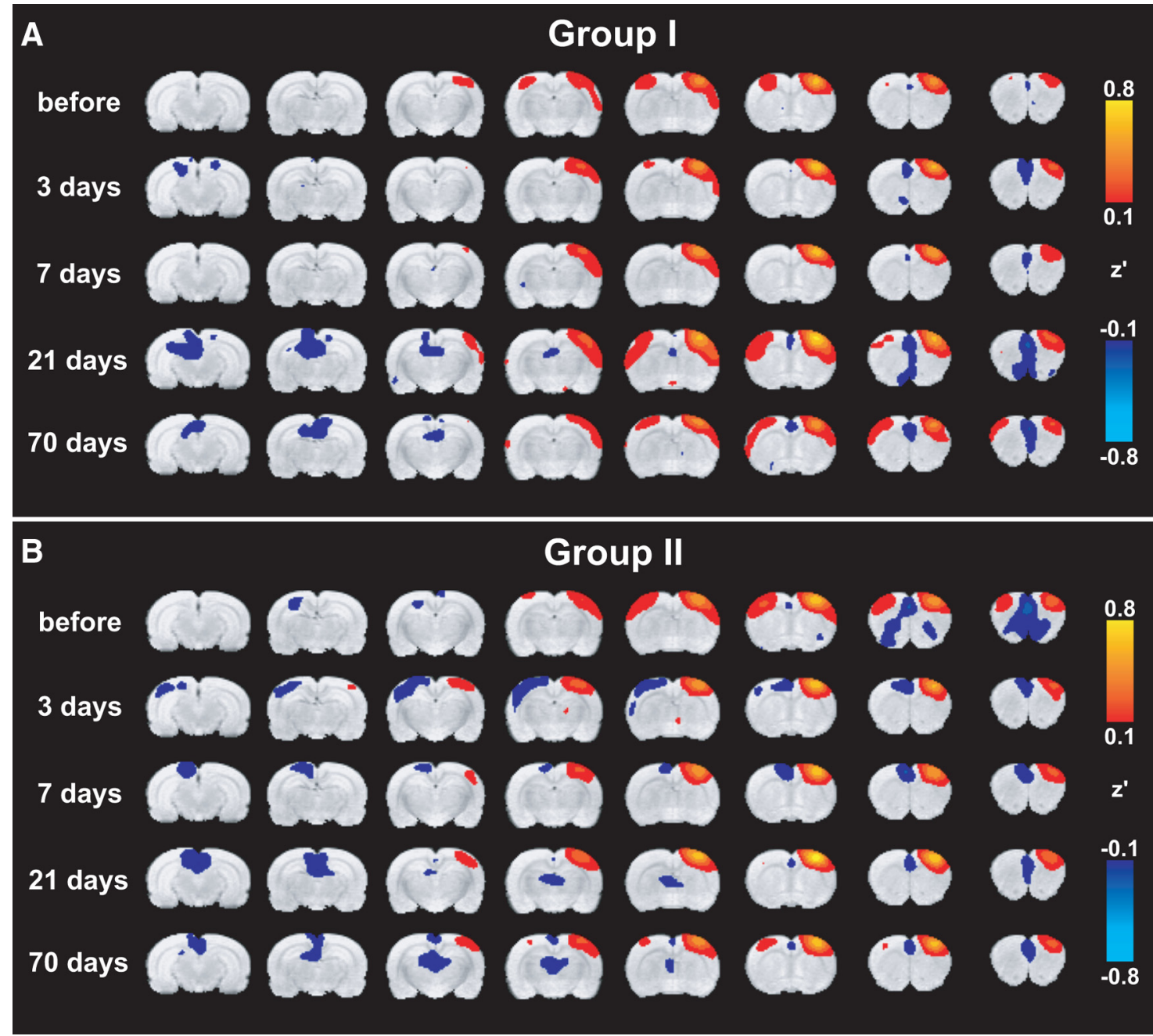

Figure 3. Functional connectivity with right S1fl. $\boldsymbol{A}, \boldsymbol{B}$, Mean functional connectivity maps of groups I $(\boldsymbol{A})$ and II $(\boldsymbol{B})$, calculated from a seed in the structurally intact right, ipsilesional $S 1 f \mid$ before and at different time points after tMCA-0. Maps display Fisher-transformed correlation coefficients $\left(z^{\prime}\right)$ ranging from 0.1 to 0.8 and -0.1 to -0.8 for positive and negative correlations, respectively, overlaid on consecutive coronal rat brain slices from a $\mathrm{T}_{2}$-weighted template.

stroke in group II (Fig. 5C). At later time points, interhemispheric functional connectivity increased but remained significantly reduced for bilateral M1 in group II up to $70 \mathrm{~d}$ after stroke.

\section{Neuroanatomical connectivity}

Administration of neuronal tracer into contralesional M1 at 10-11 weeks after stroke in a subset of group I and II animals resulted in apparent accumulation in contralesional and ipsilesional sensorimotor regions. Figure $6 A$ shows the $\Delta R_{1}$ map of a coronal brain slice of an animal from group II at $2 \mathrm{~d}$ after manganese injection. Increase of $\mathrm{R}_{1}$ because of manganese accumulation was most obvious in the contralesional sensorimotor cortex and caudate-putamen (i.e., ipsilateral to the injection site). Significant manganese transfer to the ipsilesional hemisphere was evident from the $\mathrm{R}_{1}$ increase in the $\mathrm{CC}$ and
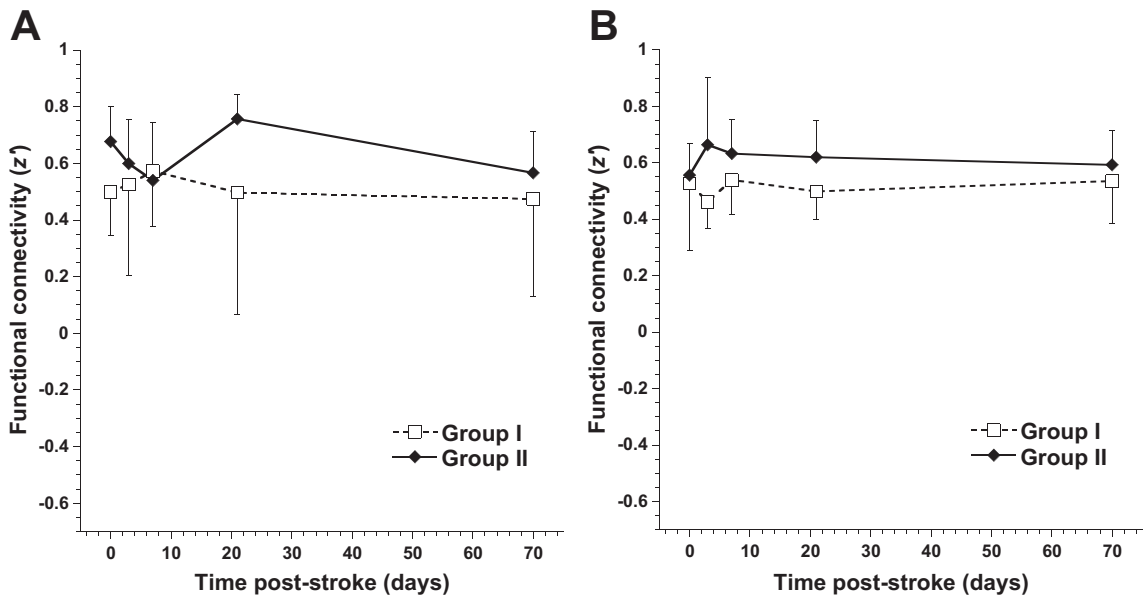

Figure 4. Intrahemispheric connectivity between S1fl and M1. $A, B$, Mean $( \pm S D)$ intrahemispheric functional connectivity $\left(z^{\prime}\right)$ between S1fl and M1, in the right ipsilesional $(\boldsymbol{A})$ and left contralesional hemisphere $(\boldsymbol{B})$, for groups I (dotted lines) and II (solid lines), before and at different time points after tMCA-0. Linear mixed-model analysis demonstrated a significantly increased functional connectivity between $\mathrm{S} 1 \mathrm{fl}$ and $\mathrm{M} 1$ in the contralesional hemisphere in group II compared with group I $(p<0.05)(B)$. 

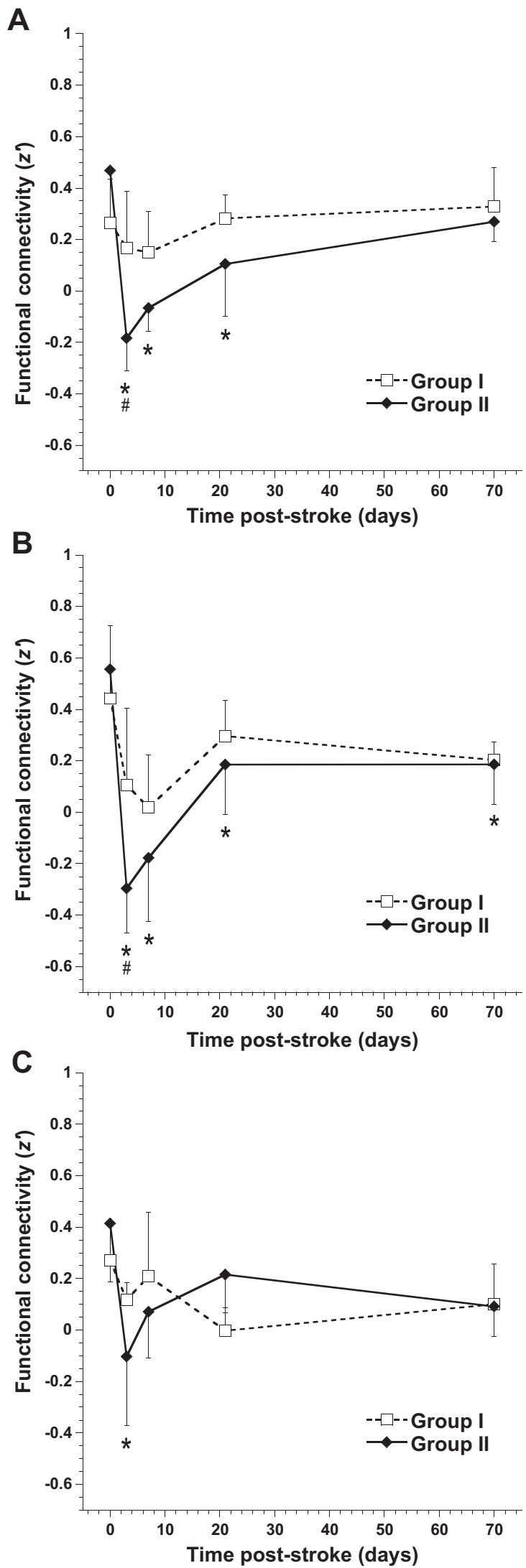

Figure 5. Interhemispheric connectivity between bilateral brain regions. $A-C$, Mean ( $\pm S D)$ interhemispheric functional connectivity $\left(z^{\prime}\right)$ between right and left S1fl $(\boldsymbol{A}), \mathrm{M} 1(\boldsymbol{B})$, and V1 $(\boldsymbol{C})$, for groups I (dotted lines) and II (solid lines), before and at different time points after tMCA-0. ${ }^{*} p<0.05$ versus pre; ${ }^{\#} p<0.05$ versus group $\mathrm{I}$. ipsilesional $\mathrm{M} 1$ and $\mathrm{S} 1 \mathrm{fl}$ in both groups (Fig. $6 \mathrm{~B}$ ). Clear $\mathrm{R}_{1}$ changes were absent inside the lesion territory. Intact transhemispheric neuroanatomical connectivity was confirmed by immunohistochemical staining for BDA and PHA-L, which demonstrated clear buildup of the anterograde tracers in the CC and ipsilesional M1 and S1fl (Fig. 6C).

\section{Correlation between sensorimotor function and functional brain connectivity}

Table 1 shows the regression coefficients and corresponding $p$ values for the correlations between the profiles of sensorimotor function scores and interhemispheric functional connectivity $\left(z^{\prime}\right)$ values per group. In both groups, the temporal pattern of changes in SPS and adhesive removal time difference (for the affected forelimb) correlated significantly with the time course of changes in interhemispheric functional connectivity between right and left S1fl, and between right and left M1. This relationship was absent for the nonsensorimotor region V1.

\section{Discussion}

In this experimental stroke study, we combined behavioral testing of sensorimotor function with rs-fMRI of functional connectivity within the sensorimotor cortical network in rats, to elucidate longitudinal transformations in bilateral neural network organization and alterations in associated functions. First, we found that sensorimotor function decline was paralleled by loss of coherence of low-frequency BOLD fluctuations in ipsilesional and contralesional primary sensorimotor cortical regions, at (sub)acute stages after stroke. This reduction of interhemispheric functional connectivity was most significant in animals with subcortical and cortical tissue damage. Yet the loss of interhemispheric functional connectivity was evident in cortical areas outside the ischemic lesion territory and also present in animals with only subcortical injury. Second, at chronic stages, sensorimotor function recovered and its temporal profile correlated significantly with changes in functional connectivity between ipsilesional and contralesional sensorimotor cortical areas. These findings suggest that synchronization of neuronal signals in bilateral homologous sensorimotor regions is associated with and may contribute to restoration of sensorimotor function in subjects recovering from unilateral cortical and/or subcortical stroke.

\section{Interhemispheric functional connectivity}

The observed (sub)acute distortion of interhemispheric functional connectivity of the ipsilesional sensorimotor cortex reflects a bilateral imbalance of synchronized spontaneous neuronal signaling, which could be explained by different stroke-induced alterations in the ipsilesional and contralesional hemisphere.

In correspondence with a disturbed functional status of ipsilesional neuronal tissue, task- and stimulus-associated fMRI studies in stroke patients and animal models have found significantly reduced activation responses in the cortical lesion border zone or ipsilesional cortex (after subcortical stroke) at early poststroke stages (Dijkhuizen et al., 2001, 2003; Tombari et al., 2004; Jaillard et al., 2005; Weber et al., 2008). Furthermore, depressed glutamatergic neurotransmitter metabolism has been measured in cortical tissue at the border of subacute ischemic lesions (van der Zijden et al., 2008b). Concurrent with loss of perilesional activity after cortical stroke, functional imaging studies have observed increases in contralesional responsiveness (Cramer and Bastings, 2000; Dijkhuizen et al., 2001, 2003; Rijntjes and Weiller, 2002; 

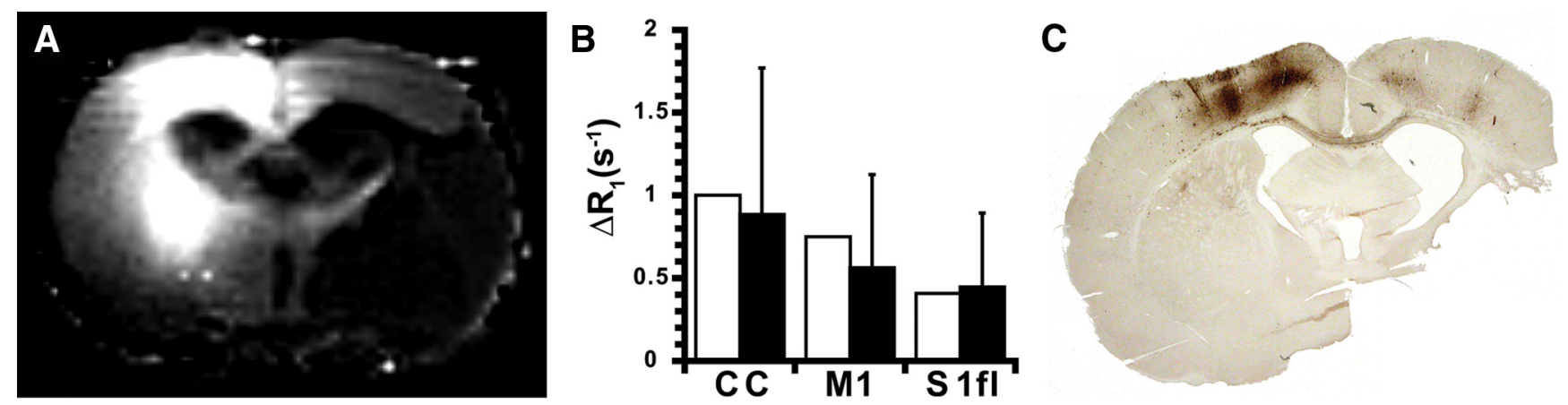

Figure 6. Neuroanatomical connectivity with contralesional $M 1$ at chronic poststroke stage. $A, \Delta R_{1}$ map of a coronal brain slice from a group II rat at $74 \mathrm{~d}$ after $t M C A-0$. Manganese-induced $R_{1}$ increase is evident in the contralesional sensorimotor cortex, contralesional caudate-putamen, corpus callosum, and ipsilesional $\mathrm{S} 1 \mathrm{fl}$ and $\mathrm{M} 1$ at $2 \mathrm{~d}$ after $\mathrm{MnCl}_{2}$ injection in the left, contralesional $\mathrm{M} 1$. $\Delta R_{1}$ was negligible inside the lesion. $B, \Delta R_{1}$ [in seconds ${ }^{-1}$; mean $( \pm S D)$ ] in $C\left(\right.$ and right, ipsilesional $M 1$ and $S 1 f l$, calculated from the difference between tissue $R_{1}$ before and $2 \mathrm{~d}$ after manganese injection in contralesional $\mathrm{M} 1$ in rats from group I (white bars) and II (black bars) at $72 \mathrm{~d}$ after stroke. C, Coronal brain section from a group II rat at $85 \mathrm{~d}$ after tMCA-0, immunohistochemically stained for BDA and PHA-L at $13 \mathrm{~d}$ after iontophoretic injections in left, contralesional M1. Staining of the anterograde tracers is clearly visible in the contralesional sensorimotor cortex, transcallosal tracts, and the ipsilesional M1 and S1fl. Remaining tissue within the lesion was detached during tissue preparation.

Table 1. Correlation between behavioral scores and interhemispheric functional connectivity

\begin{tabular}{|c|c|c|c|c|c|}
\hline \multirow[b]{2}{*}{ Sensorimotor function } & \multirow[b]{2}{*}{ Group } & \multicolumn{4}{|c|}{ Interhemispheric functional connectivity } \\
\hline & & & S1fl & M1 & V1 \\
\hline \multirow[t]{4}{*}{ SPS } & I & $r$ & 0.762 & 0.504 & 0.218 \\
\hline & & $p$ & $<0.01^{*}$ & $<0.01^{*}$ & 0.13 \\
\hline & ॥ & $r$ & 0.754 & 0.862 & 0.221 \\
\hline & & $p$ & $<0.01^{*}$ & $<0.01^{*}$ & 0.23 \\
\hline \multirow[t]{4}{*}{ Adhesive removal time difference } & 1 & $r$ & 0.909 & 0.537 & -0.010 \\
\hline & & $p$ & $<0.01^{*}$ & $0.03^{*}$ & 0.94 \\
\hline & II & $r$ & 0.755 & 0.886 & 0.153 \\
\hline & & $p$ & $<0.01^{*}$ & $<0.01^{*}$ & 0.35 \\
\hline
\end{tabular}

Standardized regression coefficients $(r)$ with corresponding $p$ values as calculated from linear mixed-model analysis of the temporal profiles of SPSs or adhesive removal time differences (for the affected left forelimb) versus the time courses of interhemispheric functional connectivity $\left(z^{\prime}\right)$ between right and left S1fl, M1, or V1.

${ }^{*} p<0.05$.

Calautti and Baron, 2003), which could be attributable to diminished GABAergic transhemispheric inhibition (Witte et al., 2000). In addition, a recent task-related fMRI study in stroke patients demonstrated that loss of normal interhemispheric balance of M1 activation during movement of the affected hand, correlated with poor motor performance (Calautti et al., 2007). Likewise, longitudinal PET and fMRI studies have shown a more physiological balance of M1 activation over time in conjunction with motor recovery (Calautti et al., 2001; Ward et al., 2003).

However, loss of excitatory input from lesioned neuronal tissue may cause attenuation of activity in intact, connected regions in the ipsilesional and contralesional hemispheres. This phenomenon is known as diaschisis and may involve all kinds of (transient) remote effects on electrical activity, blood flow, and metabolism (Feeney and Baron, 1986; Andrews, 1991). In the light of poststroke diaschisis, contralesional activity may have also been depressed and contributing to the observed reduced interhemispheric communication at early poststroke stages. In line with our findings, interhemispheric activation imbalance has recently been found in a 2-deoxyglucose autoradiography study in rats after photothrombotic stroke (Jablonka et al., 2010). In the first week after stroke, whisker stimulationinduced 2-deoxyglucose uptake was decreased in the lesioned hemisphere, while elevated in homologous areas of the contralesional cortex.

Changes in interhemispheric functional connectivity in our stroke model were not restricted to the sensorimotor system. We also detected a significant decrease of correlation between lowfrequency BOLD signals in left and right V1 in rats with subcortical and cortical ischemic injury after $3 \mathrm{~d}$. This suggests that large lesions can lead to extensive functional disconnection in the brain.

As described above, diverse ipsilesional and contralesional events can be responsible for loss of neuronal synchronization between bilateral homologous sensorimotor cortical regions after stroke. Still, at later time points, interhemispheric functional connectivity recovered, which points toward reinstatement of bilateral neuronal communication. This may reflect the disappearance of temporary interruption of synchronization through direct callosal connections, in conformity with the concept of transient transhemispheric diaschisis (Andrews, 1991). Alternatively, the resolution of asynchronous signaling of the bilateral sensorimotor regions may be related to restoration of initial unilateral disruption of the cortico-thalamo-cortical circuit. In accordance, in a previous manganese-enhanced MRI study, we have observed reestablishment of neuroanatomical connectivity of the perilesional sensorimotor cortex with subcortical regions at $>2$ weeks after tMCA-O (van der Zijden et al., 2008a). In addition, we measured increased transhemispheric corticocortical transport of manganese, which further upholds the reemergence of interhemispheric functional connectivity at chronic time points after stroke. Tracing experiments in the current study clearly demonstrated effective transfer of the neuronal tracers manganese, BDA, and PHA-L from contralesional M1 through the corpus callosum toward ipsilesional M1 and S1fl, at 10-12 weeks after tMCA-O in both experimental groups. This confirms that neuronal tissue in ipsilesional M1 and S1fl was preserved, and that transcallosal connectivity between these regions and the contralesional sensorimotor cortex was intact.

\section{Intrahemispheric functional connectivity}

In contrast to the biphasic changes in interhemispheric functional connectivity, we found no significant changes over time in intrahemispheric functional connectivity within the ipsilesional sensorimotor cortex. This seems in contradiction with abovedescribed loss of neuronal activity in this region. However, synchronization of neuronal signals may still be preserved between closely interconnected somatosensory and motor regions. Effectively, maintenance of intrahemispheric corticocortical communication could provide an important foundation for additional 
recovery of structurally intact, but functionally suppressed ipsilesional tissue.

In the contralesional hemisphere, we found an overall group effect of increased intrahemispheric connectivity between S1fl and M1 in animals with large (cortical and subcortical) lesions in the opposite hemisphere. Plastic changes in the homologous contralesional sensorimotor cortex, such as higher turnover rates of synaptic spines (Takatsuru et al., 2009) and synaptogenesis and dendritic growth (Jones et al., 1996), could lead to improved efficacy of synaptic connections. Such contralesional remodeling could play a critical role in reorganization of functional activity within bilateral neural networks and, for instance, may be related to possible active compensation with the intact forelimb during sensorimotor recovery of the affected forelimb.

\section{Correlation with behavioral recovery}

Our rs-fMRI study in rats reveals a strong correlation between sensorimotor function and the degree of interhemispheric functional connectivity of the sensorimotor cortices. Importantly, this correlation was specific for the resting-state functional connectivity of the sensorimotor network, as no correlation was found with changes in functional connectivity between the primary visual cortices. Our findings are in agreement with results from He et al. (2007), who reported initial disruption of interhemispheric functional connectivity in the structurally intact posterior parietal cortex of spatial neglect patients, which correlated with impaired attentional processing, followed by restoration at later time points. Their study and ours underline the importance of intact interhemispheric neuronal communication for improvement of behavioral outcome after cerebral injury. We speculate that this relationship is not unidirectionally causal, since improved behavior and functioning are believed to further stabilize and strengthen synaptic connections (Holtmaat and Svoboda, 2009), leading to consolidation of neuronal networks in brains recovering from stroke injury. In fact, repeated behavioral testing in our study may have contributed to the functional improvement as well as return toward more normal interhemispheric connectivity and synchronization. A recent rs-fMRI-based study on effective motor network connectivity in stroke patients demonstrated that behavioral improvement after training of the impaired upper extremity is accompanied by increased influence of the affected premotor cortex on the unaffected premotor cortex (James et al., 2009). Improved insights into the relationship between behavioral recovery and interhemispheric communication may provide support for novel therapeutic interventions aimed at rebalancing of neuronal activity across hemispheres by means of brain stimulation (Nowak et al., 2009).

\section{Limitations of rs-fMRI}

Functional connectivity measurements with rs-fMRI are based on the coherence of slow fluctuations of the BOLD signal, which is an indirect measure of synchronization of neuronal activities. It is important to note that the computed correlation strength between low-frequency BOLD oscillations in different brain regions does not inform on the degree of local neuronal activity.

Various external factors, such as $\mathrm{CO}_{2}$ (Biswal et al., 1997) and anesthesia levels (Lu et al., 2007; Vincent et al., 2007), may influence the magnitude and coherence of low-frequency BOLD fluctuations. Hence, we closely monitored expired $\mathrm{CO}_{2}$ percentage and maintained normocapnia by adjusting the ventilation rate and/or volume when necessary. Furthermore, rats were lightly anesthetized with $1 \%$ isoflurane, which preserves spatial and temporal correlations of spontaneous BOLD signal fluctuations (our unpublished data).

Respiratory and cardiac signals can also intervene with the resting-state BOLD signal in the brain (Auer, 2008). To correct for potential aliasing of physiological noise with the neuronal BOLD oscillations, we applied global mean signal regression and linear regression with signal from ventricular and white matter ROIs before correlation analysis (Weissenbacher et al., 2009). Removal of the global mean signal has been shown to enhance the detection of system-specific positive, but it also more strongly reveals negative or low correlations (Fox et al., 2009; Murphy et al., 2009; Weissenbacher et al., 2009). In our study, significant negative correlations were found between the ipsilesional sensorimotor cortex and nonsensorimotor areas, and between the ipsilesional and contralesional sensorimotor cortices, at 3 and $7 \mathrm{~d}$ after stroke in rats with relatively large lesions. Although there may be a biological basis of anticorrelated networks (Fox et al., 2009), we found no significant negative correlations when global mean signal regression was omitted (data not shown). This suggests that the observed negative correlations between brain regions most likely represent very low correlations of low-frequency BOLD fluctuations, which advocates cautious interpretation.

\section{Conclusion}

In conclusion, our rs-fMRI study in rats recovering from unilateral stroke revealed a correlation between the patterns of changes in sensorimotor function and changes in interhemispheric functional connectivity between bilateral sensorimotor cortical regions. Intrahemispheric functional connectivity between the primary somatosensory and motor cortices remained intact in the ipsilesional hemisphere, despite the significant deficits in sensorimotor function. In the contralesional hemisphere, the intrahemispheric connectivity between these regions was enhanced in animals with unilateral subcortical and cortical damage. We believe that these improvements and consolidations of intrahemispheric and interhemispheric functional connectivity in the sensorimotor cortices may play a critical role in subserving recovery of somatosensory and motor functions after stroke. Our study further demonstrates the potential of rs-fMRI to serially assess patterns of functional reorganization within neuronal networks in animal models of neurological disorders.

\section{References}

Andrews RJ (1991) Transhemispheric diaschisis. A review and comment. Stroke 22:943-949.

Auer DP (2008) Spontaneous low-frequency blood oxygenation leveldependent fluctuations and functional connectivity analysis of the "resting" brain. Magn Reson Imaging 26:1055-1064.

Biswal B, Yetkin FZ, Haughton VM, Hyde JS (1995) Functional connectivity in the motor cortex of resting human brain using echo-planar MRI. Magn Reson Med 34:537-541.

Biswal B, Hudetz AG, Yetkin FZ, Haughton VM, Hyde JS (1997) Hypercapnia reversibly suppresses low-frequency fluctuations in the human motor cortex during rest using echo-planar MRI. J Cereb Blood Flow Metab 17:301-308.

Calautti C, Baron JC (2003) Functional neuroimaging studies of motor recovery after stroke in adults: a review. Stroke 34:1553-1566.

Calautti C, Leroy F, Guincestre JY, Baron JC (2001) Dynamics of motor network overactivation after striatocapsular stroke: a longitudinal PET study using a fixed-performance paradigm. Stroke 32:2534-2542.

Calautti C, Naccarato M, Jones PS, Sharma N, Day DD, Carpenter AT, Bullmore ET, Warburton EA, Baron JC (2007) The relationship between motor deficit and hemisphere activation balance after stroke: a $3 \mathrm{~T}$ fMRI study. Neuroimage 34:322-331.

Cordes D, Haughton VM, Arfanakis K, Carew JD, Turski PA, Moritz CH, Quigley MA, Meyerand ME (2001) Frequencies contributing to func- 
tional connectivity in the cerebral cortex in "resting-state" data. AJNR Am J Neuroradiol 22:1326-1333.

Cramer SC, Bastings EP (2000) Mapping clinically relevant plasticity after stroke. Neuropharmacology 39:842-851.

Dijkhuizen RM, Berkelbach van der Sprenkel JW, Tulleken KA, Nicolay K (1997) Regional assessment of tissue oxygenation and the temporal evolution of hemodynamic parameters and water diffusion during acute focal ischemia in rat brain. Brain Res 750:161-170.

Dijkhuizen RM, Ren J, Mandeville JB, Wu O, Ozdag FM, Moskowitz MA, Rosen BR, Finklestein SP (2001) Functional magnetic resonance imaging of reorganization in rat brain after stroke. Proc Natl Acad Sci U S A 98:12766-12771.

Dijkhuizen RM, Singhal AB, Mandeville JB, Wu O, Halpern EF, Finklestein SP, Rosen BR, Lo EH (2003) Correlation between brain reorganization, ischemic damage, and neurologic status after transient focal cerebral ischemia in rats: a functional magnetic resonance imaging study. J Neurosci 23:510-517.

Dolleman-Van der Weel MJ, Wouterlood FG, Witter MP (1994) Multiple anterograde tracing, combining Phaseolus vulgaris leucoagglutinin with rhodamine- and biotin-conjugated dextran amine. J Neurosci Methods $51: 9-21$.

Engel AK, Kreiter AK, König P, Singer W (1991) Synchronization of oscillatory neuronal responses between striate and extrastriate visual cortical areas of the cat. Proc Natl Acad Sci U S A 88:6048-6052.

Feeney DM, Baron JC (1986) Diaschisis. Stroke 17:817-830.

Fox MD, Raichle ME (2007) Spontaneous fluctuations in brain activity observed with functional magnetic resonance imaging. Nat Rev Neurosci 8:700-711.

Fox MD, Zhang D, Snyder AZ, Raichle ME (2009) The global signal and observed anticorrelated resting state brain networks. J Neurophysiol 101:3270-3283.

He BJ, Snyder AZ, Vincent JL, Epstein A, Shulman GL, Corbetta M (2007) Breakdown of functional connectivity in frontoparietal networks underlies behavioral deficits in spatial neglect. Neuron 53:905-918.

He BJ, Snyder AZ, Zempel JM, Smyth MD, Raichle ME (2008) Electrophysiological correlates of the brain's intrinsic large-scale functional architecture. Proc Natl Acad Sci U S A 105:16039-16044.

Holtmaat A, Svoboda K (2009) Experience-dependent structural synaptic plasticity in the mammalian brain. Nat Rev Neurosci 10:647-658.

Honey CJ, Sporns O, Cammoun L, Gigandet X, Thiran JP, Meuli R, Hagmann P (2009) Predicting human resting-state functional connectivity from structural connectivity. Proc Natl Acad Sci U S A 106:2035-2040.

Horwitz B, Duara R, Rapoport SI (1984) Intercorrelations of glucose metabolic rates between brain regions: application to healthy males in a state of reduced sensory input. J Cereb Blood Flow Metab 4:484-499.

Jablonka JA, Burnat K, Witte OW, Kossut M (2010) Remapping of the somatosensory cortex after a photothrombotic stroke: dynamics of the compensatory reorganization. Neuroscience 165:90-100.

Jaillard A, Martin CD, Garambois K, Lebas JF, Hommel M (2005) Vicarious function within the human primary motor cortex? A longitudinal fMRI stroke study. Brain 128:1122-1138.

James GA, Lu ZL, VanMeter JW, Sathian K, Hu XP, Butler AJ (2009) Changes in resting state effective connectivity in the motor network following rehabilitation of upper extremity poststroke paresis. Top Stroke Rehabil 16:270-281.

Jones TA, Kleim JA, Greenough WT (1996) Synaptogenesis and dendritic growth in the cortex opposite unilateral sensorimotor cortex damage in adult rats: a quantitative electron microscopic examination. Brain Res 733:142-148.

Kreisel SH, Bazner H, Hennerici MG (2006) Pathophysiology of stroke rehabilitation: temporal aspects of neuro-functional recovery. Cerebrovasc Dis 21:6-17.

Laufs H, Krakow K, Sterzer P, Eger E, Beyerle A, Salek-Haddadi A, Kleinschmidt A (2003) Electroencephalographic signatures of attentional and cognitive default modes in spontaneous brain activity fluctuations at rest. Proc Natl Acad Sci U S A 100:11053-11058.

Lenz C, Rebel A, van Ackern K, Kuschinsky W, Waschke KF (1998) Local cerebral blood flow, local cerebral glucose utilization, and flowmetabolism coupling during sevoflurane versus isoflurane anesthesia in rats. Anesthesiology 89:1480-1488.

Longa EZ, Weinstein PR, Carlson S, Cummins R (1989) Reversible middle cerebral artery occlusion without craniectomy in rats. Stroke 20:84-91.

Lu H, Zuo Y, Gu H, Waltz JA, Zhan W, Scholl CA, Rea W, Yang Y, Stein EA (2007) Synchronized delta oscillations correlate with the resting-state functional MRI signal. Proc Natl Acad Sci U S A 104:18265-18269.

Maekawa T, Tommasino C, Shapiro HM, Keifer-Goodman J, Kohlenberger RW (1986) Local cerebral blood flow and glucose utilization during isoflurane anesthesia in the rat. Anesthesiology 65:144-151.

Murphy K, Birn RM, Handwerker DA, Jones TB, Bandettini PA (2009) The impact of global signal regression on resting state correlations: are anticorrelated networks introduced? Neuroimage 44:893-905.

Nowak DA, Grefkes C, Ameli M, Fink GR (2009) Interhemispheric competition after stroke: brain stimulation to enhance recovery of function of the affected hand. Neurorehabil Neural Repair 23:641-656.

Nudo RJ (1999) Recovery after damage to motor cortical areas. Curr Opin Neurobiol 9:740-747.

Pawela CP, Biswal BB, Cho YR, Kao DS, Li R, Jones SR, Schulte ML, Matloub HS, Hudetz AG, Hyde JS (2008) Resting-state functional connectivity of the rat brain. Magn Reson Med 59:1021-1029.

Paxinos G, Watson C (2005) The rat brain in stereotaxic coordinates, Ed 5. San Diego: Elsevier Academic.

Rijntjes M, Weiller C (2002) Recovery of motor and language abilities after stroke: the contribution of functional imaging. Prog Neurobiol 66:109-122.

Schallert T, Fleming SM, Leasure JL, Tillerson JL, Bland ST (2000) CNS plasticity and assessment of forelimb sensorimotor outcome in unilateral rat models of stroke, cortical ablation, parkinsonism and spinal cord injury. Neuropharmacology 39:777-787.

Shmuel A, Leopold DA (2008) Neuronal correlates of spontaneous fluctuations in fMRI signals in monkey visual cortex: implications for functional connectivity at rest. Hum Brain Mapp 29:751-761.

Takatsuru Y, Fukumoto D, Yoshitomo M, Nemoto T, Tsukada H, Nabekura J (2009) Neuronal circuit remodeling in the contralateral cortical hemisphere during functional recovery from cerebral infarction. J Neurosci 29:10081-10086.

Tombari D, Loubinoux I, Pariente J, Gerdelat A, Albucher JF, Tardy J, Cassol E, Chollet F (2004) A longitudinal fMRI study: in recovering and then in clinically stable sub-cortical stroke patients. Neuroimage 23:827-839.

van der Zijden JP, Wu O, van der Toorn A, Roeling TP, Bleys RL, Dijkhuizen RM (2007) Changes in neuronal connectivity after stroke in rats as studied by serial manganese-enhanced MRI. Neuroimage 34:1650-1657.

van der Zijden JP, Bouts MJ, Wu O, Roeling TA, Bleys RL, van der Toorn A, Dijkhuizen RM (2008a) Manganese-enhanced MRI of brain plasticity in relation to functional recovery after experimental stroke. J Cereb Blood Flow Metab 28:832-840.

van der Zijden JP, van Eijsden P, de Graaf RA, Dijkhuizen RM (2008b) ${ }^{1} \mathrm{H} /{ }^{13} \mathrm{C}$ MR spectroscopic imaging of regionally specific metabolic alterations after experimental stroke. Brain 131:2209-2219.

Vincent JL, Patel GH, Fox MD, Snyder AZ, Baker JT, Van Essen DC, Zempel JM, Snyder LH, Corbetta M, Raichle ME (2007) Intrinsic functional architecture in the anaesthetized monkey brain. Nature 447:83-86.

Ward NS, Brown MM, Thompson AJ, Frackowiak RS (2003) Neural correlates of motor recovery after stroke: a longitudinal fMRI study. Brain 126:2476-2496.

Weber R, Ramos-Cabrer P, Justicia C, Wiedermann D, Strecker C, Sprenger C, Hoehn M (2008) Early prediction of functional recovery after experimental stroke: functional magnetic resonance imaging, electrophysiology, and behavioral testing in rats. J Neurosci 28:1022-1029.

Weissenbacher A, Kasess C, Gerstl F, Lanzenberger R, Moser E, Windischberger C (2009) Correlations and anticorrelations in resting-state functional connectivity MRI: a quantitative comparison of preprocessing strategies. Neuroimage 47:1408-1416.

Witte OW, Bidmon HJ, Schiene K, Redecker C, Hagemann G (2000) Functional differentiation of multiple perilesional zones after focal cerebral ischemia. J Cereb Blood Flow Metab 20:1149-1165. 